\title{
Warehousing and Material Handling Practices in Ghana: A Tale of Tradition and Modernity
}

\author{
Mawuko Dza ${ }^{1} \&$ Evans Kyeremeh ${ }^{1}$ \\ ${ }^{1}$ School of Business, University of Education, Winneba, Ghana \\ Correspondence: Mawuko Dza, School of Business, University of Education, Winneba, South Campus, Ghana. \\ E-mail: mdza@uew.edu.gh
}

Received: June 24, 2018

Accepted: August 4, 2018 Online Published: September 19, 2018

doi:10.5539/par.v7n2p1

URL: http://dx.doi.org/10.5539/par.v7n2p1

\begin{abstract}
The paper investigates warehousing and material handling practices in the civil service of Ghana. The purpose is to ascertain the level of transition from fully manual operations to the integration of technology in warehousing and material handling operations in selected Metropolitan, Municipal and District Assemblies (MMDAs). Data were collected from 40 MMDAs cross the current 10 regions of Ghana. The study noted among others that, most warehouses lacked basic mechanical equipment for effective and efficient operations. Warehouse automation was completely non-existent in all selected Assemblies. In the case of material handling, the research discovered that very little effort has been made to equip employees with the requisite handling equipment for the execution of tasks. The study further revealed that there was virtually no in-service training on effective material handling practices in the civil service of Ghana. Indeed, practitioners most often use their bare hands and feet in handling all forms of materials including hazardous chemicals. The study also indicated that, on few instances where some handling and protective equipment were made available, most employees refused to use them citing reasons that bother on culture and tradition. The study concludes that it is imperative for authorities to put in place policies to protect practitioners in the execution of tasks. Thus, there should be an efficient change management system to ensure a gradual paradigm shift from obsolete warehousing and material handling practices to a much-integrated system where aspects of manual, mechanical and automated systems are combined.
\end{abstract}

Keywords: material handling, manual lifting, tradition, modernity, ergonomic principle

\section{Introduction}

Warehousing forms an integral part of logistics and supply chain operations. Basically, a warehouse connotes an organization's planned space used for storage and material handling purposes (Tompkins, 2010). A typical look at the supply chain from the upstream through the focal firm to the downstream indicates that, storage takes place at the various tiers from production to distribution. Warehouse management is therefore essential in ensuring a balance between production and demand. Examining warehousing from the logistics viewpoint indicates that they are facilities used to store or hold raw materials, semi-finished goods, or finished goods for varying periods (Coyle et. al., 2003). Essentially, warehouses minimize the effects of supply chain inefficiencies, improve logistics accuracy and inventory management, and allow product accumulation, consolidation, and customization. The cost of warehousing should be commensurate with the contribution of warehousing to the overall logistics performance. Although many organizations have examined the possibilities of practicing the Just-in-Time philosophy, and others looking at how effective cross-docking techniques could invalidate the significance of warehousing. The extant literature has shown that it would be very expensive, if not impossible to completely do away with warehouses. Worthy to note is the fact that, warehousing and material handling operations work hand-in-hand. The objective of material handling is to ensure efficient products and material movement, protection, storage and control in the warehouse. The concept of material handling has become important with the advent of technology integration into warehouse operations. Subsequently, some organizations operate warehouses with advanced material handling equipment. These material handling equipment increase productivity and accuracy, compared to the completely manual operations (Blanchard, 2010).

Warehousing and material handling operations has seen massive integration of technology into their operations, however, not all jurisdictions have benefitted from these technological outlays or injections. In Ghana, as in most 
emerging economies, practitioners mostly use the manual approach in executing the tasks of warehousing and material handling. This does not suggest that there have not been some levels of technological integration into warehousing and material handling in Ghana. It is however intriguing to note that, despite the provision of some basic mechanical material handling equipment for MMDAs in Ghana, tradition and culture has rendered most of the equipment redundant as practitioners invariably prefer to stick to the 'status quo', thus extensively using the traditional manual approach in executing their tasks. Significantly, research outputs in the areas of warehousing and material handling are quite substantial. It is also a fact that warehousing and material handling operations have improved across countries, including emerging economies over the past decades. These developments notwithstanding, research has shown that there is a gap in knowledge on the effect of culture and tradition on technological integration in warehousing and material handling operations in Ghana. Indeed, there is no known empirical investigation into how the tale of tradition, culture and modernity impact on warehousing and material handling operations in the MMDAs of Ghana. The objective of the research therefore is to investigate these variables and their impact on practitioners' performance in a study area known for its paucity of information.

\section{Warehousing and Material Handling Operations in Perspective}

Warehousing has become an important activity of the supply chain principally for the reason that, it is often impossible for suppliers to effectively meet the lead times required by customers, hence these customers need to be served from inventory rather than from direct order (Harrison and van Hoek, 2005). Additionally, it is prudent to hold strategic inventory at designated points in the supply chain to separate lean manufacturing activities from the downstream agile response to volatile market places (Christopher and Towill, 2001). Alternatively, supply and distribution networks may be of sufficient complexity that, there may be the need for goods to be consolidated at inventory holding points so that multi-product orders for customers can be delivered together (Higginson and Bookbinder, 2005). The operations of such warehouses are critical to the provision of high customer service levels and they need to achieve this reliably within high tolerances of speed, accuracy and lack of damage (Baker, 2004). With this critical impact on customer service levels and logistics costs, as well as the degree of complexity involved, it is thus imperative to the success of organizations that warehouses are designed to function cost effectively (Rouwenhorst et al., 2000). It is further important to assert that, because warehousing and material handling work hand-in-hand, it is imperative to properly and appropriately handle material designated for future use to ensure that they efficiently support operations for which they are meant to execute (Childe, 2003). This means that without a well-designed and functional material handling system, manufacturing operations could encounter delays; production times could increase; products could get damaged or contaminated; and cost of movement within facilities could increase, thereby increasing operating cost. On the other hand, a well thought-out and planned material handling system could help manufacturing and logistics facilities improve their productivity, enhance products' quality, and reduce operating costs (Hassan, 2006).

Indeed, in order to promote effective and efficient material handling operations, the Material Handling Institute (MHI, 2000), has proposed what has become known as the "Ten Material Handling Principles". These principles are stated and explained as follows: Planning. According to the planning principle, all material handling operations should be the result of a well thought-out plan where the needs, performance objectives, and functional representation of the proposed methods are completely designed at the outset. Standardization. The standardization principle states that material handling methods, equipment, controls and software should be standardized within the limits of achieving overall performance objectives and without sacrificing needed flexibility, modularity and throughput. Work. The work principle suggests that, material-handling tasks should be minimized without sacrificing productivity or the level of service required of the operation. Ergonomic. The ergonomic principle arguably one of the most important, stresses that human capabilities and limitations must be recognized and respected in the design of material handling tasks and equipment to ensure safe and effective operations. Unit Load. The objective of the Unit load principle is to ensure that unit loads are appropriately sized and configured in a way that achieves the material flow and inventory objectives at each stage in the supply chain. Space Utilization. Effective and efficient use must be made of all available space. The key points of this principle are that, in work areas, cluttered and unorganized spaces and blocked aisles should be eliminated. On storage, the objective of maximizing storage density must be balanced against accessibility and selectivity. Further, the principle suggests that when transporting loads within a facility the use of overhead space should be considered as an option. System. The system principle emphasizes that material movement and storage activities should be fully integrated to form a coordinated, operational system which spans receiving, inspection, storage, production, assembly, packaging, unitizing, order selection, shipping, transportation and the handling of returns. Automation. The automation principle advocates that material handling operations should be mechanized and/or automated where feasible to improve operational efficiency, increase responsiveness, improve consistency and 
predictability, decrease operating costs and eliminate repetitive or potentially unsafe manual labour. Environmental. Environmental impact and energy consumption should be considered as criteria when designing or selecting alternative equipment and material handling systems. This ensures that very little or no harm is done to the environment during material handling operations. Life Cycle Cost. A thorough economic analysis should account for the entire life cycle of all material handling equipment and resulting systems (MHI, 2000).

These "Ten Key Material Handling principles" seek to transform the handling process in ensuring safety, efficiency and optimization in manufacturing/production. Effective compliance to the principles for instance could ensure that heavy loads are properly and appropriately handled to minimize practitioner injury; large volumes of different items are handled without damage or loss thereby saving costs; and order picking, packing and inventory tracking would be done with little or no errors. In order for these propositions come to fruition, manufacturers and users must be careful when designing and implementing new systems that seek to enhance efficiency in material handling (Furmans, et. al., 2010).

\section{Integrating Technology into Warehousing and Material Handling Operations}

The rapid evolution of warehousing and material handling operations especially in the area of technological integration makes it imperative for organisations to either adapt to modern trends of doing business or risk being crowded out. Technologies like Warehouse Management Systems (WMS), Radio Frequency Identification (RFID), Automated Guided Vehicle System (AGVS) among others, have transformed and revolutionalised the landscape of warehousing and material handling across parts of globe. For instance the RFID uses radio waves to exchange data between a reader and electronic tags attached on objects. The data on the tags can be read and written to facilitate the identification and tracking of the objects in warehouses (Chen et. al., 2013). The RFID has attracted significant attention in the fields of logistics and supply chain because of its effectiveness and efficiency (Hunt et al., 2007) in tracking goods throughout the supply chain and also as one of the new technologies influencing the operations in production, warehousing, and distribution sectors (Chen et. al., 2013). The AGVS is a material handling system that uses independently operated, self-propelled vehicles guided along defined pathways in the facility floor. It is an automated material handling system, which moves along predefined and pre-programmed path along an aisle from one station to another. The main parts of an AGVS include structure; drive system, steering mechanism, power source (battery) and onboard computer for control (Aized, 2006).

Research has shown that, the WMS and RFID technology are integrated at three levels thus, data collection, data movement and data management (Tan, 2008). According to Tompkins \& Smith (1998), a computerized WMS represents a tool that can facilitate the automation and optimization of material handling processes; improve inventory accuracy and facility usage; reduce labor costs; and enhance order-picking accuracy. A WMS is therefore a key part of logistics and supply chains as it aims at controlling the movement and storage of materials within warehouses. The system also directs and optimizes stock putaway based on real-time information about the status of bin utilization (Arora, 2009). The objective of a WMS is therefore to provide a set of computerized procedures to handle the receipt of stock and returns into a warehouse facility; model and manage the logical representation of the physical storage facilities; manage the stock within the facility and enable a seamless link to order processing and logistics management (Arora, 2009). It is significant to acknowledge that, an automated warehouse forms an integral part of sustainable warehousing because it often reduces operational costs. A thoughtfully planned automated system leaves little or no wastes. All types of goods maintained in a warehouse or distribution center are carried out through automation systems with the assistance of conveyors and overhead pulley systems (Garcia et. al., 2003). Computer systems are placed in strategic locations to facilitate the movement of these goods from bulk storage area to the next activity areas of the warehouse. It is further justified to state that current developments in material handling operations are leading to automated systems that move faster, accommodate greater throughput, and require less maintenance (García, et. al., 2003).

\section{Manual and Mechanical Material Handling Operations}

According to the University of California's DAVIS Safety Services (2015), material handling is classified as manual when it involves the use of absolute physical labour in lifting, lowering, and carrying objects. It is important to indicate that, in manual handling if ergonomic principles are ignored, there is a high probability of strain on the muscles, joints, and disks in the back which could eventually lead to injury. For this reason, it is advised that objects that are too heavy or bulky for safe manual handling by employees should be lifted and moved by mechanical lifting devices. Moving raw materials and finished products through a facility is a common process in the manufacturing industry. Throughout that process, operators with various manufacturing tasks routinely lift/lower, push/pull and carry objects, where risk factors sometimes leading to musculoskeletal 
disorders (MSDs) may occur (Batish \& Singh, 2008). It is imperative to stress that, although technology has advanced industrial production techniques, manual handling of materials have remained essentially the same. Most jobs require some manual handling, but about 10 per cent require extensive manual material handling (Texas Department of Insurance, Division of Workers' Compensation (TDI, DWC, 2009). While emphasizing that manual material handling is required in most operations, it is significant to stress that sometimes a material handling operations could be executed either completely manual or automated. The level of mechanization is classified with respect to the degree of practitioner involvement and computer applications in operating equipment. With regards to mechanized operations, mechanical means instead of physical effort is used for driving the equipment. Trucks, conveyors, and cranes fall into this level. Accordingly, operators are needed for operating the equipment as opposed to using physical strength. As the degree of mechanization increases, there is system complexity, which results in improved efficiency but increases design and operating costs (Groover, 2007). Mechanized material-handling equipment is mostly used by organizations to attain higher efficiency and improved productivity at a relatively lower cost. Although mechanical handling creates a new set of hazards, the net result is fewer injuries, lower workers' compensation expenses, and a more productive workplace (TDI, DWC, 2009).

\section{Warehousing and Material Handling Hazards}

In 2017, the National Safety Council (NSC) of the United States reports that warehousing and material handling accounts for $20 \%$ to $45 \%$ of all work related injuries. Further, research has shown that the most susceptible parts of the body prone to warehousing and material handling injuries are the human toes and fingers. Based on this evidence, the NSC proposes that it is imperative for practitioners and handlers to wear protective accouterment in order to minimize the risk of injuries that occur at every facet of operations (NSC, 2017). Further, the Occupational Safety and Health Administration (OSHA), postulates that warehousing and material handling practitioners suffer from excessive back injuries. These back injuries according to the OSHA, accounts for almost $20 \%$ of workplace injuries, which results from lifting, lowering, pushing, pulling, carrying material and twisting the body. Experts put the annual cost of back injuries at between $\$ 20$ billion and $\$ 50$ billion US dollars. While acknowledging weight a major cause of back injuries, it is prudent to stress that weight is not the only factor that causes back problems. Other factors include: bending, stretching, handling large and awkward loads, carrying loads over long distances or for long periods of time, among others (TDI, DWC, 2009). Frazer (2003), reports that, practitioners or handlers who exceed the maximum permissible limit (MPL) are at risk of increased musculoskeletal injury rates. This conclusion is grounded on the basis that in reality only about $25 \%$ men and a little below $1 \%$ women workers have the muscle strength to be capable of performing work above the MPL (Seo, 2016). Another important cause of warehousing and material handling injuries is the use of inexperienced or untested employees. This happens because of their low levels of experience and lack of practical expertise to handle various demands and techniques of tasks assigned to them. Although training is a major component of injury management and prevention, it is important that the work environments as well as working conditions are improved in order to reduce the risk of workplace injuries. According to the National Institutes of Occupational Safety and Health (NIOSH), "the best way to protect workers is through ergonomic approaches designed to reduce the hazards of lifting". This new line of thinking incorporates advanced ergonomic trends in warehousing and manual material handling operations (TDI, DWC, 2009).

\section{The Influence of Culture and Tradition on Institutional Performance}

Warehousing and Material Handling are activities that have been performed for centuries. Invariably, haven transcended various traditions and cultures, to what is now known as the modern phase of the profession, research suggests that the influence of culture and tradition in their operations cannot be overstressed. Indeed, the naive assumption that management and work ethics is the same or is becoming the same around the world as a result of modernity is untenable in view of demonstrated differences in national cultures (Dza, 2013). This debate is particularly important in this era of global operations, as organisations need to know whether management controls that are effective in one national setting will have alternate levels of effectiveness if used in a different national setting (Chow, Shields, and $\mathrm{Wu}, 1999)$. Research has shown that national cultural values are related to workplace behaviours, attitudes and other organizational outcomes (Trompenaars, 1993). Culture is a characteristic of individuals, and it encompasses a number of people who are conditioned by the same education and life experience. Culture in this sense of collective mental programming, is often difficult to change, and if it does change at all, it does so slowly. The change is slow not only because it exists in the minds of the people but, because it has become crystalized in the institutions these people have built together (Hofstede, 1983). 


\section{Data Collection and Methodology}

The study is a cross-sectional descriptive survey employing both the qualitative and quantitative methods of data collection and analysis. Questionnaires and structured interview guides were the main primary sources used to collect data from participants who after thorough explanation of the importance of the study, willingly agreed and signed "informed consent forms". Data collected were analysed and presented by means of descriptive techniques of analysis. Further analysis was performed (inferential statistical analysis) which made use of correlation, hypothesis testing and multiple linear regression analysis.

\section{Data Analysis and Discussions}

Data were conveniently collected from 40 District and Municipal Assemblies across the current 10 regions of Ghana. As indicated earlier, the study adopted primarily the descriptive techniques of analysis and inferential statistical analysis for further justification of results. The descriptive analysis comprises of mean and percentage and analysis on correlation, hypothesis testing and multiple linear regression analysis for the inferential analysis.

Below is the modeling equation on regression analysis:

$$
\begin{aligned}
& y=\tau+\varphi_{1} x_{1}+\varphi_{2} x_{2}+\varphi_{3} x_{3}+\ldots+\varphi_{n} x_{n}+\gamma_{i} \\
& E(\bar{y})=\tau+\varphi_{1} x_{1}+\varphi_{2} x_{2}+\varphi_{3} x_{3}+\ldots+\varphi_{n} x_{n}
\end{aligned}
$$

Where, $E(\bar{y})$ is the mean responses variable; $\tau$ is the mean coefficient of the regression constants; $\varphi_{1}, \varphi_{2}, \varphi_{3}, \ldots, \varphi_{n}$ are the means coefficients of the predictor variables and; $x_{1}, x_{2}, x_{3}, \ldots, x_{n}$ the means of the predictors.

Table 1. Statistical analysis on warehouse and material handling methods

\begin{tabular}{cccccc}
\hline Material Handling & Mean & Percentage & Average weight of Materials in Warehouse & Mean & Percentage \\
\hline Manual & 4.61 & $79.9 \%$ & $\leq 20 \mathrm{~kg}$ & 1.21 & $2.9 \%$ \\
Mechanical & 1.16 & $20.1 \%$ & $\leq 50 \mathrm{~kg}$ & 2.35 & $33.3 \%$ \\
& & & $>50 \mathrm{~kg}$ & 4.50 & $63.7 \%$ \\
Total & & $\mathbf{1 0 0}$ & Total & & $\mathbf{1 0 0}$ \\
\hline
\end{tabular}

Significant at the level (alpha $=0.05),(2$-tailed)

From table 1, the average rate of materials handled manually stands at 4.61(79.9\%). This implies that almost $80 \%$ of materials are handled using the traditional manual approach. The table also gives an indication that the use of mechanical handling equipment such as the hand forklifts, pallet jacks, conveyor belts, mini cranes among others remain minimal, thus about $20 \%$ (1.16). It was further observed that, the average weight of materials handled manually is $20 \mathrm{~kg}$ whilst those handled mechanically had average weight of $50 \mathrm{~kg}$. The ever-increasing use of the manual and basic mechanical equipment in material handling contradicts the study by Blanchard (2010), whose research findings indicates that integrating technology into material handling operations has the propensity to increase productivity and accuracy as compared to the traditional manual system. It is important to affirm that the use of manual handling approach expose workers to myriads of injuries. Indeed, according to the United States' NCS report in 2017, most occupational injuries happened as a result of manually handling materials. Despite this worrying statistics, it is important to state that, mechanical handling cannot absolve itself from workplace injuries, as research has shown that injuries do occur even with the use of mechanical equipment to handle material. The difference is that, there are much fewer injuries in using mechanical handling techniques as opposed to manual handling operations (TDI, DWC, 2009).

It is significant to acknowledge that all MMDAs that constituted the basis for this research had neither automated warehouses nor automated material handling systems. Majority of respondents when questioned on why they depended on the manual method of operation indicated that it was a matter of choice. They explained that the manual system was more convenient as it causes less damage to stocks. Respondents further explained that it 
was much easier to locate items in the warehouse when done manually as compared to mechanical means. When quizzed on whether they knew the consequences of manually lifting heavy weights and handling hazardous chemicals, their response was that: "this is a generational thing, our forefathers used it and nothing happened to them. It's just about taking the right precautions". When asked about whether they knew the dangers of improperly carrying heavy items to designated locations, participants' responses clearly exposed their ignorance on the dangers of improperly lifting items manually. Most participants indicated that: "as for heavy loads, once you can lift them, it no longer posses a threat. If you cannot lift the item alone, then you get some colleagues to assist you move it to its designated location in the warehouse". These findings are consistent with Trompenaars' (1993) study which findings suggest that, national cultural values are related to workplace behaviours, attitudes and other organizational outcomes.

On the use of technology to improve work performance and increase productivity, the research participants suggested that: "resources that the government would use to bring in modern technology should be channeled to other sectors of the economy, as we are very satisfied with what we have". Participants also bemoan the fact that technology deployment would require long periods of training, which according to some of them would amount to waste of time since they may not eventually understand the concepts, more so use them appropriately. This stance is affront to the immense contributions technologies such as the WMS, RFID and AGVS are making towards revolutionalizing the landscape of warehousing and material handling operations to ensure improved productivity as a result of the increased effectiveness and efficiency that they bring along (Chen et. al., 2013). A careful analysis of participants' reluctance to embrace new approaches of doing things goes to buttress the point about how difficult it is for people to change from the status quo (business as usual). Participants' behaviour supports Hofstede's (1983) research findings on the effect of institutional culture on performance. The reluctance to change maybe the result of the fact that their work ethics has become so crystalized or engrained in the institutions these people have built together and transferred the knowledge to generations over the years.

Table 2. Correlation of factors between tradition and modernity

\begin{tabular}{|c|c|c|c|c|c|c|}
\hline \multicolumn{7}{|c|}{ Modernity } \\
\hline & & $\varphi_{1}$ & $\varphi_{2}$ & $\varphi_{3}$ & $\varphi_{4}$ & $\varphi_{5}$ \\
\hline \multirow{5}{*}{ 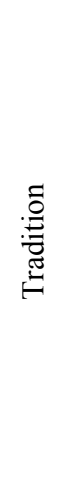 } & $\varphi_{1}$ & 1 & & & & \\
\hline & $\varphi_{2}$ & $.310^{* *}$ & 1 & & & \\
\hline & $\varphi_{3}$ & $.291^{* *}$ & $.263^{* *}$ & 1 & & \\
\hline & $\varphi_{4}$ & $.320^{* *}$ & $.331^{* *}$ & $.341^{* *}$ & 1 & \\
\hline & $\varphi_{5}$ & $.283^{* *}$ & $.301^{* *}$ & $.250^{* *}$ & $276^{* *}$ & 1 \\
\hline
\end{tabular}

\footnotetext{
** Correlation is significant at the level (2-tailed)
}

From table 2, the pairs of factors $\left(\varphi_{i}, \varphi_{j}\right)$ have correlation coefficients of 0.200 to 0.360 . Since the correlation coefficients between the manual method and modern methods (automated system) fall between 0.200 and 0.360 , multi-collinearity posses no problem for this data. There is a weak correlation between the manual method and the modern approach (automated system). The correlation of factors shows that the manual method is not dependent on modernity (automation). This goes to further explain the assertion that most employees choose the manual method because of ease of work, as they do not understand the operations of automated systems, which in any case are not available to them for use. From the analysis, it is clear that man-hours and improved 
productivity could be lost because of the nature of operations. For instance, if Tompkins \& Smith's (1998) research conclusion is anything to go by, then it stands to reason that a computerized WMS can improve inventory accuracy and facility usage, reduce labor costs, and enhance order-picking accuracy. It is also significant to stress that most material handling and warehouse injuries are caused by either manual operation. For instance, research records show that handling of material accounts for $20 \%$ to $45 \%$ of all occupational injuries. Strains, sprains, fractures, and contusions are the most common forms of injuries resulting from manually handling equipment. Most back injuries come from tasks requiring lifting (TDI, DWC, 2009). Further interrogation of research participants revealed that virtually no training in given to material handling employees. They however explained that for employees manning the warehouses, they have acquired some level of theoretical tutelage whilst undertaking their formal education. The research participants were quick to point out that, there is hardly any in-service training organized for them. The lack of training especially for material handling employees expose them to many forms of dangers. Indeed, a major cause of material handling dangers is the use of inexperienced or untested employees who have a higher probability of accidents because they are new to the job (TDI, DWC, 2009).

\section{Hypothesis Testing}

$\mathrm{H}_{\circ}$ : There is no significant difference between automated systems and the manual method to material handling.

$\mathrm{H}_{a}$ : There is significant difference between automated systems and the manual method to material handling.

Table 3. Model summary

\begin{tabular}{ccccc}
\hline Model & $\mathrm{R}$ & R square & Adjusted R square & Std. Error of the Estimation \\
\hline 1 & $\mathbf{. 8 7 3 1}^{a}$ & $\mathbf{. 6 9 5 1}$ & .7521 & .0510 \\
\hline
\end{tabular}

a. Dependent variable: automated system

b. Predictors: (constant), manual method

Analysis of table 3, indicates the coefficient of determination to be $0.5371(53.71 \%)$ of the variation between automated system and the traditional manual method of material handling as explained by the regression model. $46.29 \%$ of the variation could not be accounted for. This is as a result of culture, old methods, and general work ethics, lack of in-service training and perhaps non-usage of technology into material handling and warehouse operations.

Table 4. Analysis of variance (ANOVA)

\begin{tabular}{lllllc}
\hline Model & Sum of Squares & Df & Means of Squares & F & Sig. \\
\hline Regression & 4.666 & 9 & .518 & 10.156 & $.0000^{b}$ \\
Residuals & 1.219 & 30 & .051 & & \\
Total & 5.885 & 39 & & & \\
\hline
\end{tabular}

a. Dependent variable: traditional manual method

b. Predictors: (constant), automated system

The analysis of variance (ANOVA) table above shows that Test Statistic $(F=10.156)$ and the p-value $(p=0.000)$ are significant. Since $[p$-value $<0.000 \leq 0.05]$, we have enough evidence to reject the null 
hypothesis $\left(\mathrm{H}_{\circ}\right)$. Hence, we conclude at $\alpha=5 \%$ level of significance, implying that, there exist enough evidence that, there is significant difference between the manual method and automated systems of material handling and warehousing.

\section{Conclusion}

The paper on "Warehousing and Material Handling Practice in Ghana: a tale of tradition and modernity" sought to investigate the extent to which culture and tradition has either positively or negatively impacted the adoption of technologies such as WMS, RFID, AGVS, and advanced mechanical equipment in warehousing and material handling operations in the public sector of Ghana. The findings revealed that, warehouse automation is non-existent in all MMDAs sampled; a clear indication that, not much has been achieved as far as automating warehouse operations in the country is concerned. This phenomenon is regrettable, as research has indicated the immense contributions of automated systems to effectiveness and efficiency of warehousing and material handling operations. The study further demonstrates how culture and tradition has impacted rather negatively on warehousing and material handling operations in the public service. Interestingly, although some employees express the desire of using mechanical and automated handling systems as well as other protective equipment, the majority is opposed to the idea arguing that the fully manual approach is most suitable for handling objects because, apart from the strains, materials are delivered intact to designated locations. This is explained in the context that even where there was basic mechanical equipment for material handling operations, most practitioners preferred to use manual methods as according to them; it is much convenient and efficient. Indeed, the researchers are not oblivious of the fact that the manual approach would continue to form an integral part of warehousing and material handling, as currently between $10 \%$ and $20 \%$ of materials are handled manually. What is perhaps worrying is the non-adherence to ergonomic principles where human capabilities and limitations are not recognized and respected in the design of material handling tasks to ensure safety and cost efficient operations. Violations of this principle are resulting in various forms of injuries to practitioners. Investigations also indicated that besides the academic qualification of practitioners, there is virtually no in service training for practitioners on modern trends in warehousing and material handling techniques. However, training may not be necessary as most practitioners consider it as a waste of state resources, which according to them could be channeled to other sectors of the economy. To these practitioners, maintaining the status quo, thus sticking to manual warehousing and material handling is a sure way to improve efficiency in operations. Impliedly, this means that current and successive employees would receive on-the-job training from their predecessors.

It is intriguing to observe the lack of enthusiasm and gross apathy by practitioners towards embracing modern techniques of efficiently managing warehouses and handling materials. Surprisingly they seem unperturbed about the propensity of occupational hazards that occur daily during operations. They also seem unconcerned about the long-term effects of their physical conditions as a result of persistently lifting heavy weights, falls, and exposure to hazardous chemicals, among others. Since culture and tradition manifest in the "way a group of people do things", it has become obvious that the work ethics of warehousing and material handling practitioners may have been influenced by culture and tradition, making it difficult to change "a crystalized mindset".

In conclusion, it is quite obvious that a combination of myriad factors may be responsible for the current state of warehousing and material handling operations in MMDAs in Ghana. The influence of culture and tradition has undoubtedly played their role in slowing the integration of technology into the operation of warehousing and material handling, as it has become difficult to change the status quo. On the other hand, authorities either lack the political will to transform the sector or are constrained by resources in carrying out reforms in the sector. Whatever the reason, the bottom line is that not much change has been seen and experienced in warehousing and material handling in MMDAs across the country.

\section{References}

Aized, T. (2006). Materials handling in flexible manufacturing systems. Department of Mechanical, Mechatronics and Manufacturing Engineering, KSK, Lahore.

Arora, P. (2009). Material management. Global India Publications.

Baker, P. (2004). Aligning distribution center operations to supply chain strategy. International Journal of Logistics Management, 15(1), 111-123. https://doi.org/10.1108/09574090410700266

Batish, A. T., \& Singh P. (2008). An Assessment Tool for Analysing Manual Material Handling Tasks. International Journal of Occupational Safety and Ergonomics (JOSE), 14(2), 223-235. 
https://doi.org/10.1080/10803548.2008.11076754

Blanchard, D. (2010). Supply chain management best practices. Hoboken, N.J: John Wiley \& Sons.

Chen, J. C., Cheng, C. H., Huang, P. B., Wang, K. J., Huang, C. J., \& Ting, T. C. (2013). Warehouse management with lean and RFID application: a case study. The International Journal of Advanced Manufacturing Technology, 69(1-4), 531-542. https://doi.org/10.1007/s00170-013-5016-8

Childe, S. J. (2003). Six things to manage equipment. Production Planning \& Control, 14(7), 587. https://doi.org/10.1080/09537280310001649921

Chow, C. W., Shields, M. D., \& Wu, A. (1999). The importance of national culture in the design of and preference for management controls for multi-national operations. Accounting, Organizations and Society, 24(5), 441-461. https://doi.org/10.1016/S0361-3682(99)00047-1

Christopher, M., \& Towill, D. (2001). An integrated model for the design of agile supply chains. International Journal of Physical Distribution \& Logistics Management, 31(4), 235-246. https://doi.org/10.1108/09600030110394914

Coyle, J. J., Bardi, E. J., \& Langley, C. J. Jr. (2003). The Management of Business Logistics: A Supply Chain Perspective (7th ed.). South-Western Publishing, Mason, $\mathrm{OH}$.

Dza, M. (2013). Applying Service-Dominant Logic into the Procurement Function in Ghanaian Universities. Doctoral dissertation, Griffith University.

Furmans, K., Schonung, F., \& Gue, K. R. (2010). Plug-and-work material handling systems In Proceedings of the international material handling research colloquium.

García, A., McFarlane, D., Fletcher, M., \& Thorne, A. (2003) Auto-ID in materials handling. White paper, Auto-ID Center, Cambridge, MA, USA.

Groover, M. P. (2007). Fundamentals of modern manufacturing: materials processes, and systems. John Wiley \& Sons.

Harrison, A., \& van Hoek, R. (2005). Logistics Management and Strategy (2nd ed.). Pearson, Harlow.

Hassan, M. M. D. (2006). Engineering supply chains as systems. Systems Engineering, 9(1), 73-89. https://doi.org/10.1002/sys.20042

Higginson, J. K., \& Bookbinder, J. H. (2005). Distribution centres in supply chain operations. In A. L. Langevin, \& D. Riopel (Eds.), Logistics Systems: Design and Optimization (pp. 67-91). Springer, New York. https://doi.org/10.1007/0-387-24977-X_3

Hofstede, G. (1983). Cultural Constraints in Management Theories. Academy of Management Executive, 7, 8194.

Hunt, V. D., Puglia, M., \& Puglia, A. (2007). A guide to radio frequency identification. John Wiley \& Sons Inc. https://doi.org/10.1002/0470112255

MHI. (2000). The Ten Principles of Material Handling. Charlotte, NC: Material Handling Institute. Retrieved from http://www.mhi.org/downloads/learning/cicmhe/guidelines/10_principles.pdf

National Institutes of Occupational Safety and Health (NIOSH). Material Handling and Control by National Safety Council. Retrieved July 20, 2018, from https://docmh.com

National Safety Council (NSC). (2017). Accident Prevention Manual for Business and Industry, Engineering \& Technology (13th ed.). University of Central Missouri, USA. Retrieved July 20, 2018, from https://www.slideshare.net/complianceandsafety/materials-handling-and-storage-by-nsc

Occupational Safety and Health Administration (OSHA). Back Disorders and Injuries. Retrieved July 20, 2018, from https://www.osha.gov/dts/osta/otm/otm_vii/otm_vii_1.html

Rouwenhorst, B., Reuter, B., Stockrahm, V., van Houtum, G., Mantel, R., \& Zijm, W. (2000). Warehouse design and control: framework and literature review. European Journal of Operational Research, 122(3), 515-533. https://doi.org/10.1016/S0377-2217(99)00020-X

Seo, J. (2016). Evaluation of Construction Workers Physical Demands Through Computer Vision-Based Kinematic Data Collection and Analysis.

Tan, H. (2008). The application of RFID technology in the warehouse management information system, Proceedings of the International Symposium on Electronic Commerce and Security, Guangzhou, China, 
1063-1067.

Texas Department of Insurance, Division of Workers' Compensation. (2009). Manual Material Handling: An Ergonomic Approach.

Thompkins, J. A., \& Smith, J. D. (1998). The Warehouse Management Handbook (2nd ed.). North Carolina: Thompkins Press.

Tompkins, J. A. (2010). Facilities planning. Hoboken, NJ: John Wiley \& Sons.

Trompenaars, F. (1993). Riding the Waves of Culture: Understanding Cultural Diversity in Business, 6-10. London: Nicholas Brealey.

University of California DAVIS Safety Services. (2015). Manual Material Handling Ergonomic. Retrieved July 20, 2018, from https://safetyservices.ucdavis.edu/article/manual-material-handling-ergonomics

\section{Copyrights}

Copyright for this article is retained by the author(s), with first publication rights granted to the journal.

This is an open-access article distributed under the terms and conditions of the Creative Commons Attribution license (http://creativecommons.org/licenses/by/4.0/). 